\title{
Morphological abnormalities in lymphocyte mitochondria associated with iron-deficiency anaemia
}

\author{
J. H. JARVIS AND A. JACOBS \\ From the Department of Haematology, Welsh National School of Medicine, Heath Park, Cardiff
}

SYNOPSIS Morphological abnormalities in lymphocyte mitochondria of iron-deficient subjects are described. The abnormalities are of a degenerative type and are similar to those described in the mitochondria of other cells during iron deficiency.

A variety of tissue changes have been described in subjects suffering from iron-deficiency anaemia, and although previously it had been generally assumed that these resulted from a derangement of intracellular metabolism, no satisfactory correlation between decreased iron-enzyme activity and morphological or functional abnormalities have been found in the mouth, pharynx, stomach, or small intestine (Jacobs, 1969). Structural changes have been demonstrated in the mitochondria of liver (Dallman and Goodman, 1971) and myocardium (Goodman, Warshaw, and Dallman, 1970) in iron-deficient rats. The abnormalities included enlarged, rounded, and electron-lucent mitochondria in the hepatocyte and an enlargement of mitochondria in heart muscle, possibly with an increase in number. A similar abnormality has been observed in erythroblast mitochondria from the bone marrow of human irondeficient subjects (Dallman and Goodman, 1971).

Iron deficiency produces pathological changes in both human lymphocytes and polymorphonuclear leucocytes. Joynson, Jacobs, Murray, Walker, and Dolby (1972) demonstrated a defect of cell-mediated immunity in peripheral blood lymphocytes from patients with iron-deficiency anaemia, and Chandra (1973) has demonstrated that polymorphs from irondeficient patients have a reduced bactericidal capacity. Since iron deficiency is known to affect both the structure and iron-enzyme activity of the mitochondria in some tissues the present preliminary study was undertaken to investigate the ultrastructure of mitochondria in peripheral blood lymphocytes from iron-deficient patients.

Received for publication 5 September 1974.

\section{Methods}

Six healthy adult members of the hospital staff (controls) and six patients with untreated irondeficiency anaemia were studied. The haematological status of the two groups is shown in the table.

\begin{tabular}{lcc}
\hline & Controls & Iron Deficient \\
\hline Haemoglobin $(\mathrm{g} / 100 \mathrm{ml})$ & $14.4 \pm 0.44$ & $8.0 \pm 0.76$ \\
MCV $(\mathrm{f})$ & $\mathbf{8 8 . 2} \pm 1.01$ & $\mathbf{6 6 . 8} \pm \mathbf{2 . 7 0}$ \\
Serum-iron $(\mu \mathrm{g} / 100 \mathrm{ml})$ & $\mathbf{9 2 . 6} \pm \mathbf{3 . 8 0}$ & $32.7 \pm 5 \cdot 10$ \\
T.I.B.C. $(\mu \mathrm{g} / 100 \mathrm{ml})$ & $318 \pm 13.40$ & $\mathbf{4 8 9} \pm 16.80$ \\
\hline
\end{tabular}

Table Haematological status of the two groups $($ mean $\pm S E M)$

Peripheral blood leucocytes were prepared for electron microscopic examination by a modification of the procedure described by Anderson (1965). Twenty-five to $30 \mathrm{ml}$ of blood was collected into heparinized tubes ( 5 units heparin/ml), and then transferred to narrow centrifuge tubes (diameter $5 \mathrm{~mm}$ ). The blood was centrifuged at $300 \mathrm{~g}$ for two min to achieve swift separation into layers without cell damage. The small diameter of the centrifuge tube resulted in the formation of a 'buffy coat' 1-2 mm thick. Without disturbing the 'buffy coat' the supernatant plasma was removed and replaced with cacodylate-buffered glutaraldehyde $(2.5 \%$ glutaraldehyde in $0 \cdot 1 \mathrm{M}$ sodium cacodylate at $\mathrm{pH}$ 7.4). The cells were fixed in this solution for one $h$ at $4^{\circ} \mathrm{C}$. In addition to fixing the leucocytes, the glutaraldehyde causes the plasma between them to solidify so that the 'buffy coat' can be cut into small pieces and handled as blocks of tissue during subsequent processing. The cells were postfixed in $1 \%$ 
osmium tetroxide in $0 \cdot 1 \mathrm{M}$ sodium cacodylate (pH 7.4) at $4^{\circ} \mathrm{C}$ for one $\mathrm{h}$, dehydrated in graded ethanols and embedded in araldite. Thin sections were cut on an LKB ultramicrotome and those with grey or silver interference colours (ie, $400-800 \mathrm{~A}^{\circ}$ thick) were mounted on uncoated copper grids. The sections were stained with uranyl acetate $(30 \mathrm{~min})$ followed by lead citrate $(15 \mathrm{~min})$, and viewed with a Philips 300 electron microscope. The cells were fixed in the glutaraldehyde $\mathbf{2 0}$ min after removal of blood from the subject and the subsequent processing was rigidly standardized in all cases.

Figs 1-3 Electronmicrographs of lymphocytes from normal human peripheral blood. Glutaraldehyde/osmium fixed.

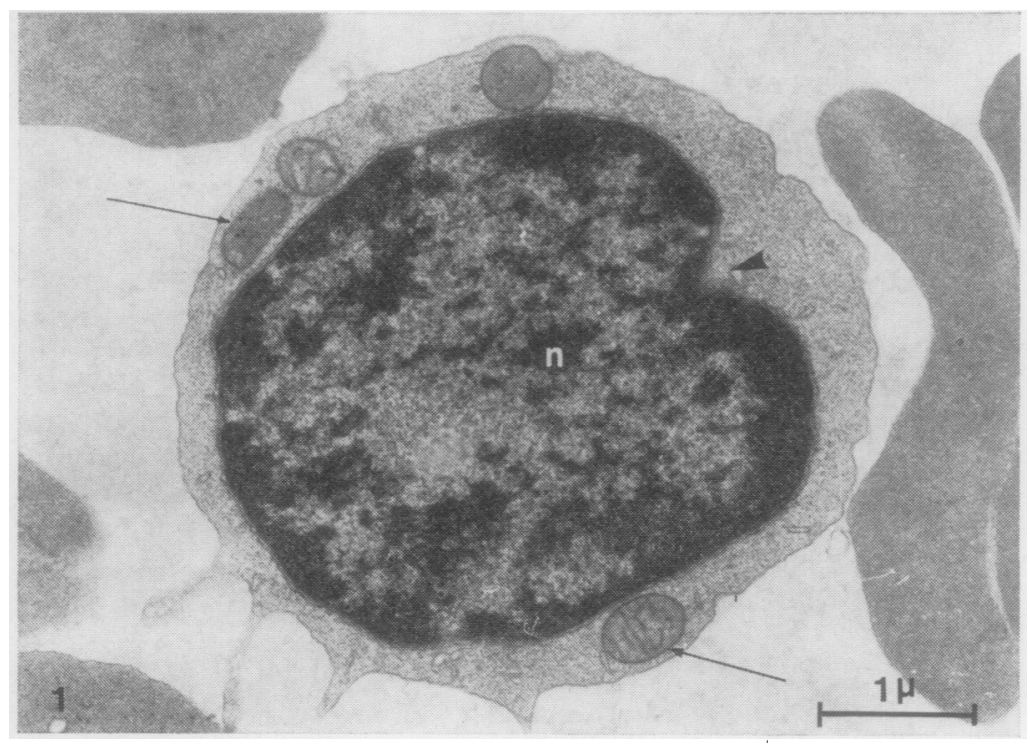

Fig 1 Electronmicrograph of small lymphocyte with characteristically large nucleus $(n)$ with indentation (arrowhead), and narrow rim of peripheral cytoplasm containing several mitochondria (arrows).

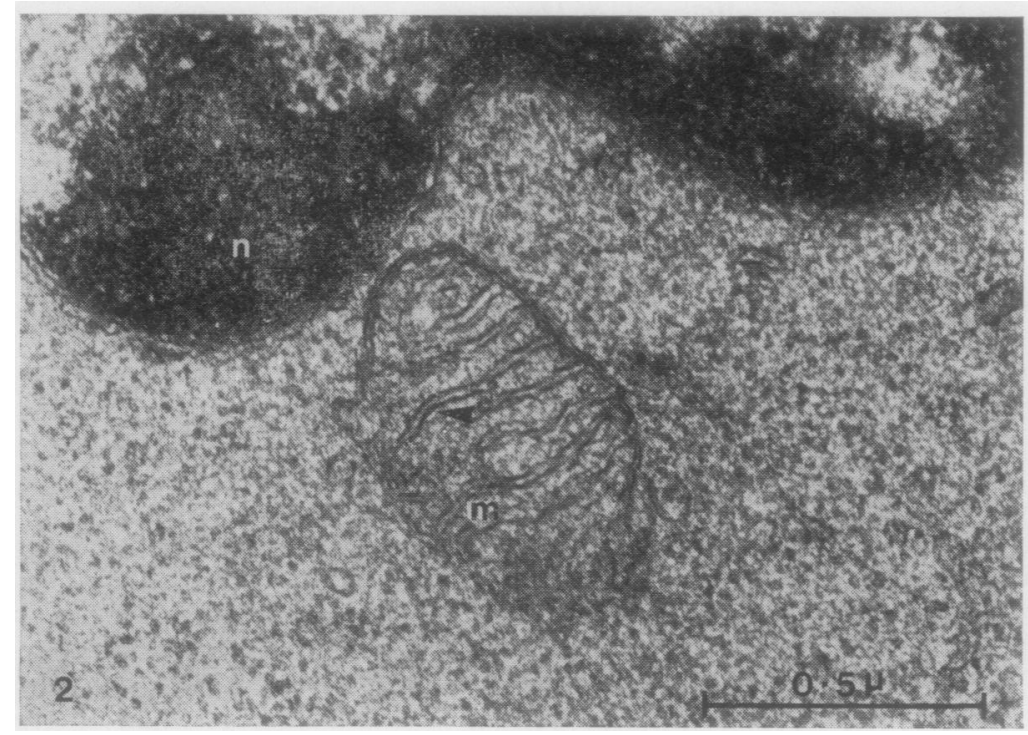

Fig 2 Electronmicrograph of tangentially sectioned mitochondrion $(m)$ adjacent to nucleus (n). Cristae (arrowhead) are well defined and the mitochondrial matrix and cytoplasm are of similar electron density. 


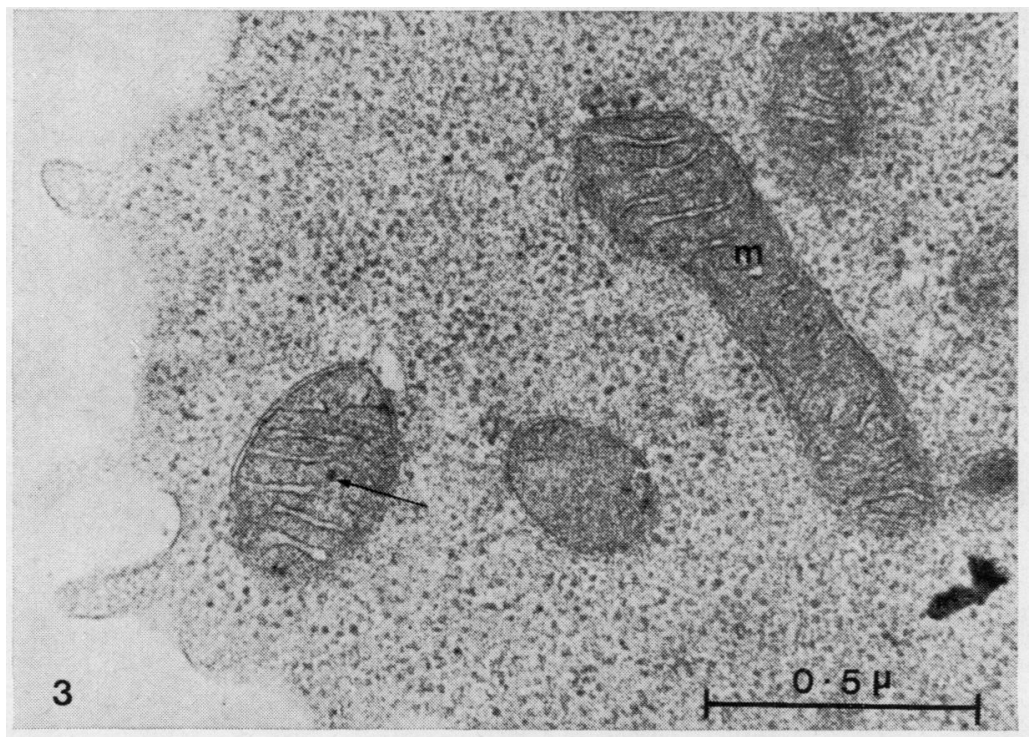

Fig 3 Electronmicrograph of mitochondria $(m)$ in peripheral lymphocyte cytoplasm. One mitochondrion contains intramitochondrial granules (arrow).

\section{Results}

Mitochondria were seen in many, but not all, sections (fig 1). Their shape was variable but they were usually spherical in transverse section, oval when sectioned tangentially (fig 2 ), and filamentous in longitudinal section (fig 3). The outer and inner mitochondrial membranes were clearly definable with distinct cristae, some of which passed from one wall of the mitochondrion to the other (figs 2 and 3 ). The density of the mitochondrial matrix was virtually constant in all the controls, usually being the same as that of the surrounding cytoplasm (fig 2). Occasionally cells were seen where the matrix was

Figs 4-9 Electronmicrographs of peripheral blood lymphocytes from subjects with iron-deficiency anaemia. Glutaraldehyde/osmium fixed.

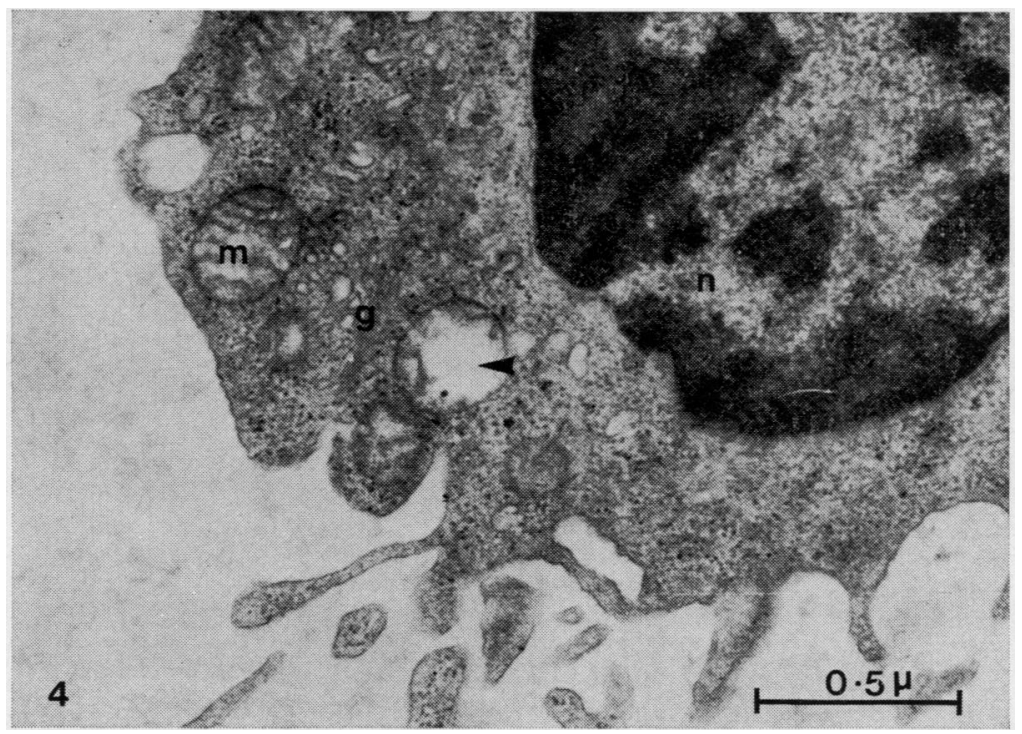

Fig 4 Electronmicrograph of lymphocyte cytoplasm adjacent to nucleus $(\boldsymbol{n})$. Several cytoplasmic organelles are apparent; a mitochondrion (m) with fairly electronlucent matrix, Golgi complex $(g)$ and an almost completely vacuolated mitochondrion (arrowhead). 


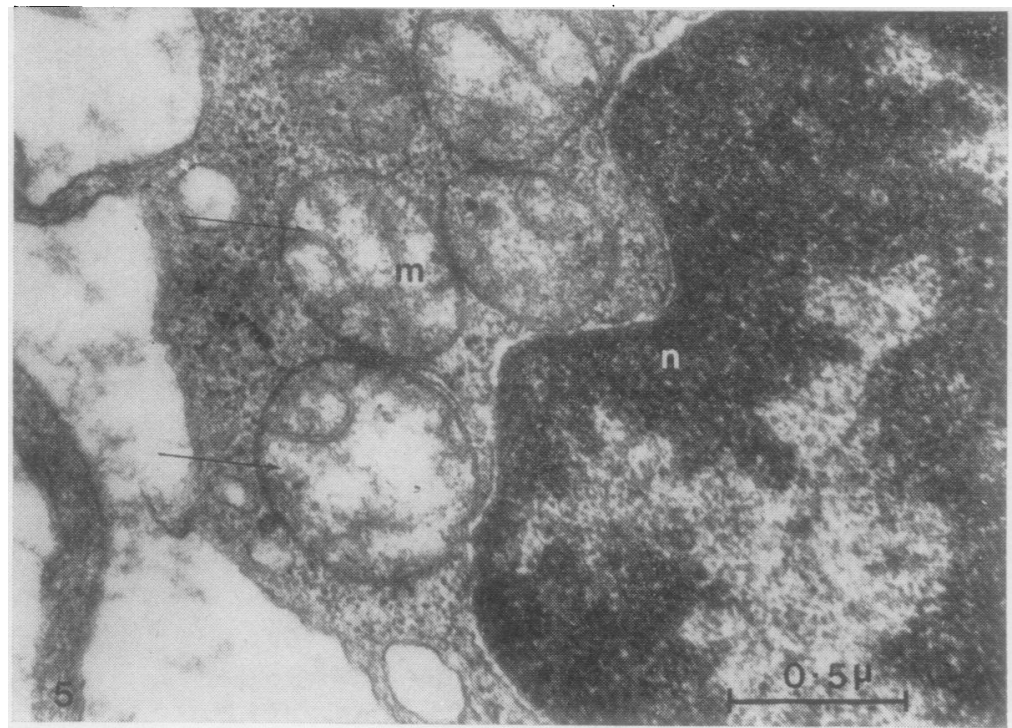

Fig 5 Electronmicrograph of mitochondrial cluster $(m)$, each showing various degrees of vacuolation. Only small fragments of cristae remain (arrows).

slightly denser than the cytoplasm (fig 3). Intramitochondrial granules, which are thought to be binding sites for divalent cations such as $\mathrm{Ca}^{++}$, were seen in some sections (fig 3).

Marked changes were observed in the lymphocyte mitochondria of all six iron-deficient patients (figs 4-9). In four of these the changes were similar to hose described in hepatocytes from iron-deficient rats. Mitochondria were swollen and showed various stages of vacuolation (figs 4-7). This vacuolation was initially obvious as a more electron-lucent matrix followed by a progressive breakdown of cristae and reduction in number until only one or two were apparent in the degenerating organelle (fig 5). After the disintegration of all cristae the mitochondrior had the appearance of an electron-lucent vacuole but since the vacuolation did not occur in all mito chondria of a cell at the same time, such vacuolated mitochondria often occurred adjacent to mitochondria of more normal appearance (fig 6). In some mitochondria the vacuolation occurred in discrete areas of the matrix so that one part of the mito-

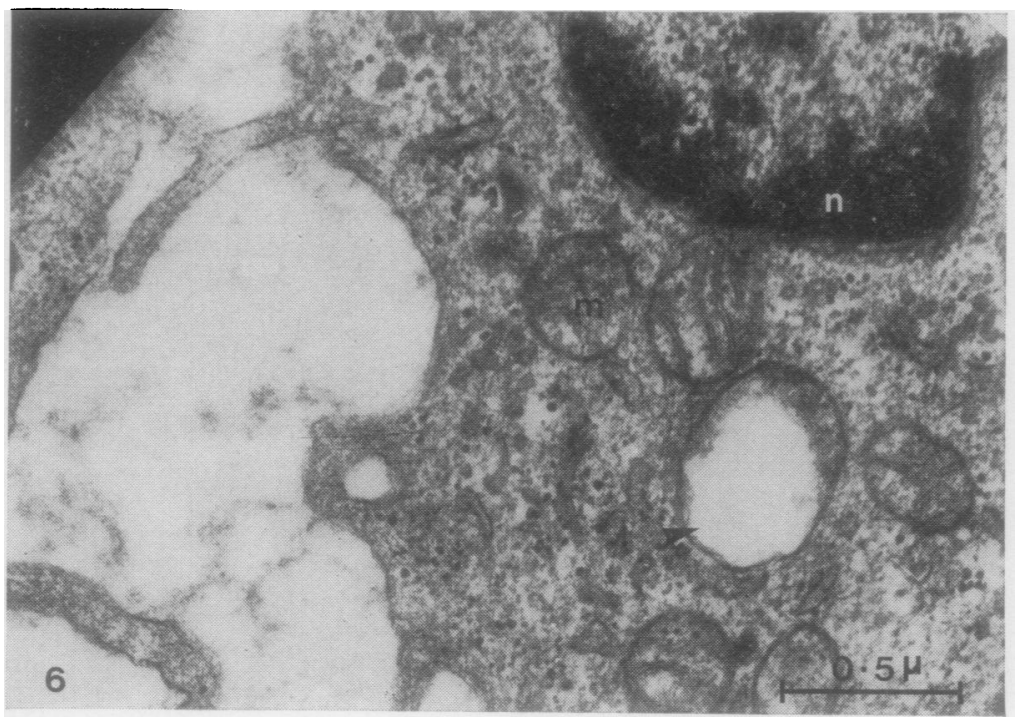

Fig 6 Electronmicrograph of mitochondria adjacent to nucleus (n). A mitochondrion with cristae $(m)$ is visible alongside a vacuolated mitochondria with no visible cristae (arrowhead). 


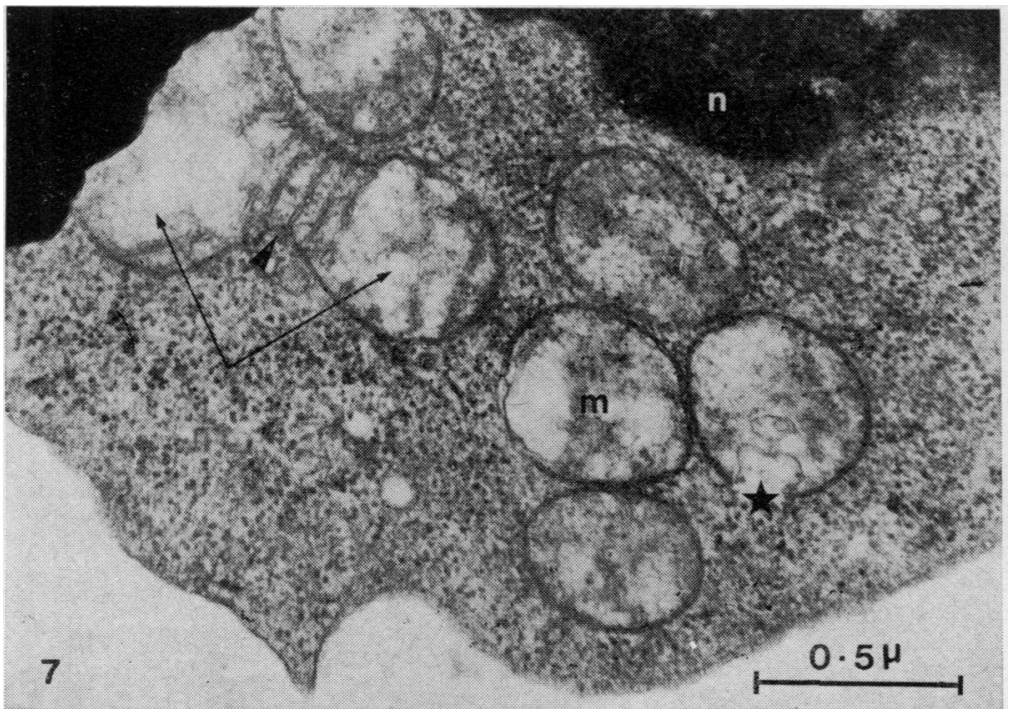

Fig 7 Electronmicrograph of mitochondria adjacent to nucleus $(n)$. The mitochondria $(m)$ are in various stages of vacuolation with no cristae discernible. In one mitochondrion vacuolation has occurred in discrete areas (arrows) whereas cristae can be seen in another part of the same organelle (arrowhead). In one mitochondrion there is evidence of the breakdown of the outer membrane (star).

chondrion retained normal dimensions and crista structure whereas another part was swollen with few, if any, cristae (fig 7). The nuclei of these abnormal cells were morphologically the same as those in control cells, but the cytoplasm of some contained a few small smooth membraned vesicles (figs 4 and 6). Golgi complexes were seen in some sections (fig 4). The outer membrane of vacuolated mitochondria usually remained intact, but there was evidence of its breakdown in some cells (fig 7).

The lymphocytes of the remaining two iron- deficient patients showed similar nuclear and cytoplasmic features to those of the four previously described patients, but in addition some cells had large electron-dense inclusions (fig 8). The most constant morphological feature of the mitochondria of these cells was the greatly increased electron density of the matrix, and the expansion of the cristae (figs 8 and 9), which in many cells led to the appearance of cristae of heterogenous shape many being triangular (fig 9). The cytoplasm of some cells contained occasional polyribosomes (fig 9) and

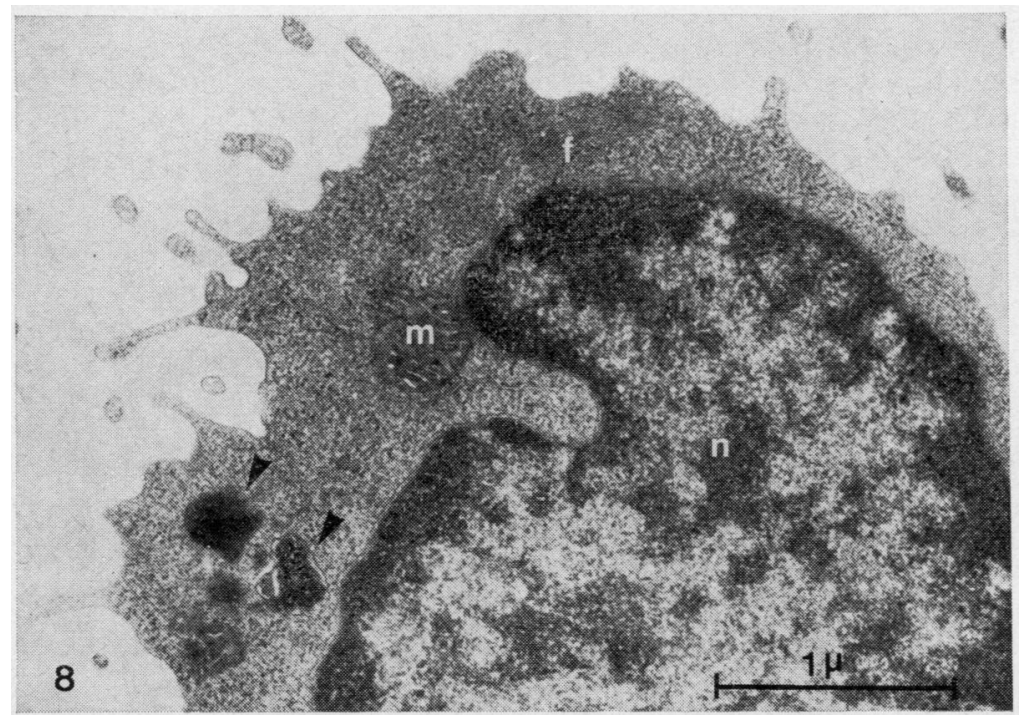

Fig 8 Electronmicrograph of cytoplasm adjacent to nucleus (n). Cytoplasmic inclusions include a mitochondrion $(m)$ with electrondense matrix, electron-dense vesicles (arrowheads), and stacks of fibrillar material $(f)$. 


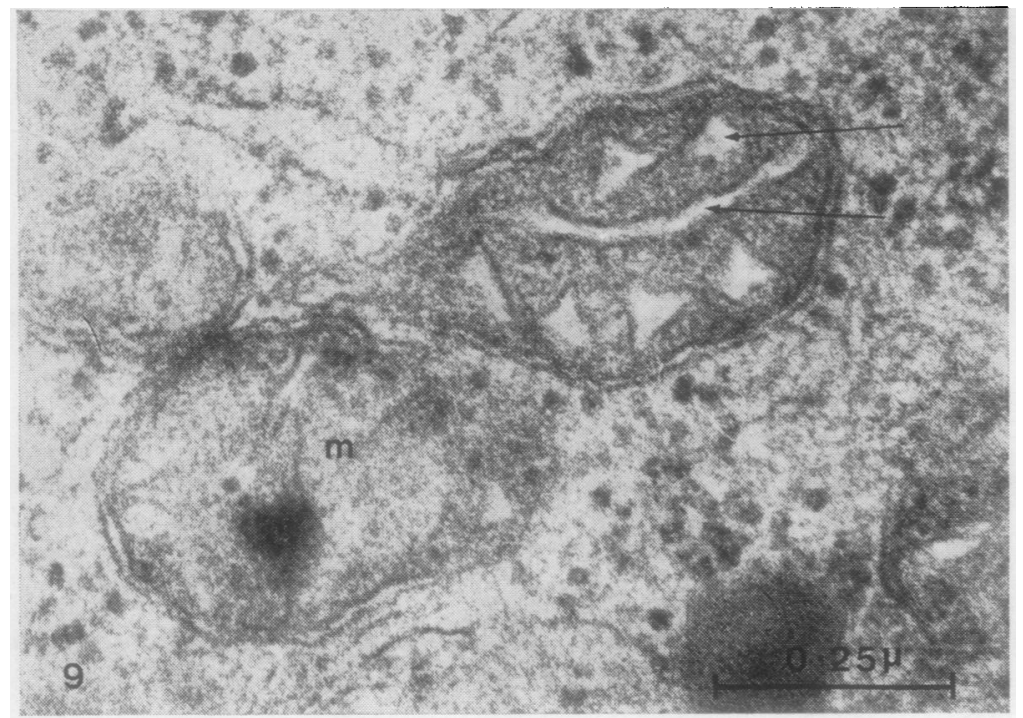

Fig 9 Electronmicrograph of mitochondria $(m)$ in lymphocyte cytoplasm. Expanded cristae are triangular and one is parallel to long axis of mitochondrion (arrows).

stacks of those peculiar fibrils recently described in other cell types (fig 8). The frequency of these fibrils has been shown to be higher in cells of patients with severe or chronic illnesses (Zucker-Franklin, 1969).

\section{Discussion}

Mitochondrial abnormalities are one of the most sensitive indicators of injury to the cell (Roodyn and Wilkie, 1968) and show a spectrum of morphological modifications as a result of pathological conditions. The mitochondrial morphology of normal cells varies from one cell type to another, but is more or less constant in cells of a similar type having the same function. In addition to the various structural differences between mitochondria of normal cells, there are well defined degenerative changes which may occur under adverse conditions. Essentially there are three types of change (De Robertis, Nowinski, and Saez, 1965). Mitochondria may swell with breakdown of cristae and transformation into vacuoles, accumulate material in the matrix leading to increased electron density, eg, lipid accumulation in mitochondria of aging cells, or fuse with other mitochondria to form large bodies called chondriospheres. The first type of degenerative change occurs in hepatocyte mitochondria of iron-deficient rats (Dallman and Goodman, 1971), and occurred in lymphocyte mitochondria of four of the irondeficient patients in this investigation. As in rat hepatic tissue, not all mitochondria of one cell, or necessarily all cells of the same tissue, show degenerative changes, but they were present in over
$90 \%$ of the lymphocytes. The swelling is distinguish- $\frac{3}{3}$ able from that due to inadequate fixation where $\frac{\mathbb{Q}}{-}$ adjacent mitochondria invariably show a similar $\vec{\theta}$ degree of structural damage. The possibility that the morphological changes seen in iron-deficient cefs resulted from a fixation artifact was considered unlikely in the present study because of the use of $\supset$ buffered fixative at physiological $\mathrm{pH}$, and by the rigid standardization of cell processing in all speci- $\frac{\circ}{\circ}$ mens of controls and patients.

The density of the matrix is influenced by the metabolic state of the mitochondrion (Tandler and Hoppel, 1972). Mitochondria swell to a slight degree when their respiration rate is slow and the ratelimiting component is ADP concentration. Some shrinking of mitochondria occurs when the respir- atory rate is slow or zero due to low substrate 3 .

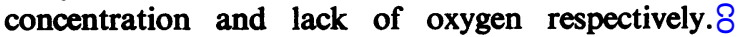
Mitochondria with a high respiratory rate may also be slightly shrunken, the respiration rate-limiting 을 component in this case being the capacity of the respiratory chain. The possibility that the increasedo density of the mitochondrial matrix observed in two of the iron-deficient patients may be at least partiallyo due to their metabolic state cannot be discounted, $N$ but the uniformity of their appearance and degree of N electron density in all leucocytes, together with the marked abnormalities in shape of cristae seems to constitute a genuine degenerative change in this organelle associated with iron deficiency.

References
Anderson, D. R. (1965). A method of preparing peripheral leucocytes for electron microscopy. J. ultrastruct. Res., 13, 263-268. 
Chandra, R. K. (1973). Reduced bactericidal capacity of polymorphs in iron deficiency. Arch. Dis. Childh., 48, 864-866.

Dallman, P. R., and Goodman, J. R. (1971). The effects of iron deficiency on the hepatocyte: a biochemical and ultrastructural study. J. Cell Biol., 48, 79-90.

Goodman, J. R., Warshaw, J. B., and Dallman, P. R. (1970). Cardiac hypertrophy in rats with iron and copper deficiency: quantitative contribution of mitochondrial enlargement. Pediat. Res., $4,244-256$.

Jacobs, A. (1969). Tissue changes in iron deficiency. Brit. J. Haemat., 16, 1-4.

Joynson, D. H. M., Jacobs, A., Murray Walker, D., and Dolby, A. E.
(1972). Defect of cell-mediated immunty in patients with iron deficiency anaemia. Lancet, 2, 1058-1059.

De Robertis, E. D. P., Nowinski, W. W., and Saez, F. A. (1965). In Cell Biology, 4th ed., pp. 166-185. Saunders, Philadelphia and London.

Roodyn, D. B., and Wilkie, D. (1968). The Biogenesis of Mitochondria. Methuen's Monographs on Biological Subjects). Methuen, London.

Tandler, B., and Hoppel, C. L. (1972). Mitochondria (Ultrastructure of Cells and Organisms, edited by Michael Locke). Academic Press, New York and London.

Zucker-Franklin, D. (1969). The ultrastructure of lymphocytes. Sem. Hemat., 6, 4-27. 